\title{
IOT BASED HOME AUTOMATION USING RASPBERRY PI
}

\author{
Trideep Singha Roy, Soumalya Ghosh, Rimpi Datta, Arpita Santra \\ ECE, Narula Institute of Technology, Kolkata, India
}

\begin{abstract}
This paper presents the design of the low cost home automation system using the IoT(Internet of Things) technology along with the feature of speech recognition. The Internet of things (IoT) is the inter-networking of physical devices, vehicles, buildings, and other items embedded with electronics, software, sensors, actuators, and network connectivity that enable these objects to collect and exchange data. In this project IoT technology is used to control the home appliances wirelessly over the internet. The computing module used is a Raspberry pi development board. The project also aims to provide a speech control interface to the users to control the appliances. Speech recognition is provided using an online Speech-To-Text platform called wit. The home automation system listens for the user's speech and whenever a defined phrase is identified it triggers corresponding action to switch appliances on or off. With speech recognition physically challenged people can control appliances with much more ease
\end{abstract}

Key words: IoT, Raspberry pi, Speech recognition.

Cite this Article: Trideep Singha Roy, Soumalya Ghosh, Rimpi Datta, Arpita Santra, IoT Based Home Automation Using Raspberry PI, International Journal of Computer Engineering and Technology 10(3), 2019, pp. 70-74.

http://iaeme.com/Home/issue/IJCET?Volume=10\&Issue=3

\section{INTRODUCTION}

Today people are looking at ways and means to better their life-style using the latest technologies that area available. Any new facility or hope appliance that promises to enhance their life-style is grabbed by the consumers. The more such facilities and appliances are added, it becomes inevitable to have easy and convenient methods and means to control and operate these appliances. Conventional wall switches are located in different parts of a house and thus necessitates manual operations like to switch on or off these switches to control various appliances. It gets virtually impossible to keep track of appliances that are running and also to monitor their performances.

Installing, arming, disarming, paying monthly fees, and dealing with false alarms are all things that are tied to monitored security systems which may cause you to wonder if home security systems are worth the inherent hassle. This is a good question to ask as you consider how you can best protect your valuables and your loved ones. There are a lot of elements to think about when it comes to home security systems and that includes how the security 
system could benefit you and your family. Here are a few reasons how that is true.This is, of course, the benefit most people immediately think of. We likely all know someone who has lost electronics, jewelry, or other high-value items due to a home invasion. The tragedy is compounded when the item is an irreplaceable family heirloom. A home security system has an alarm that scares off many would-be burglars and can notify the local authorities if someone does attempt a break-in. A 2009 study by Rutgers found that, as the number of home security systems increased in an area, the number of residential robberies decreased in that area, even for people who didn't have their own security system. Having a security system not only protects you, but helps your neighborhood be a safer place for everyone. Modern security systems now allow you to remotely monitor what's happening in your home when you're not there. Depending on your provider, you can monitor what happens via cameras installed throughout your home, as well as control the thermostat, door locks, lights, and other devices in your home. Many security systems offer smart thermostats and outlets. If you've forgotten to change your thermostat before leaving on a trip, these gadgets allow you to control the thermostat on any web-enabled device [5]. Likewise, if you think you left your curling iron plugged in, you can turn it off right from any of your devices. This works as a great tool to turn lights on and off while you're on vacation to help give the home the appearance that someone is there. Adding a security system to your home gives you an extra layer of defense against any potential intruders. Whether you rent or own, you can enjoy the benefits that come with having a security system. Explore your options using the SafeWise security system finder, which helps you to identify your security needs and goals, and find the right provider to meet them. [6]

\section{HARDWARE COMPONENTS REQUIRED:}

A Raspberry $\mathrm{Pi}$ is a credit card-sized computer originally designed for education, inspired by the 1981 BBC Micro. Creator Eben Upton's goal was to create a low-cost device that would improve programming skills and hardware understanding at the pre-university level. But thanks to its small size and accessible price, it was quickly adopted by tinkerers, makers, and electronics enthusiasts for projects that require more than a basic microcontroller (such as Arduino devices). The Raspberry Pi is slower than a modern laptop or desktop but is still a complete Linux computer and can provide all the expected abilities that implies, at a lowpower consumption level. Fig 6 raspberry pi The Raspberry Pi is open hardware, with the exception of the primary chip on the Raspberry Pi, the Broadcomm SoC (System on a Chip), which runs many of the main components of the board-CPU, graphics, memory, the USB controller, etc. Many of the projects made with a Raspberry Pi are open and well-documented as well and are things. We have built and modify these. There are a two Raspberry Pi models, the $\mathrm{A}$ and the $\mathrm{B}$, named after the aforementioned $\mathrm{BBC}$ Micro, which was also released in a Model A and a Model B. The A comes with 256MB of RAM and one USB port. It is cheaper and uses less power than the B. The current model B comes with a second USB port, an Ethernet port for connection to a network, and 512MB of RAM. The Raspberry Pi A and B boards been upgraded to the $\mathrm{A}+$ and $\mathrm{B}+$ respectively. These upgrades make minor improvements, such as an increased number of USB ports and improved power consumption, particularly in the $\mathrm{B}+$. 


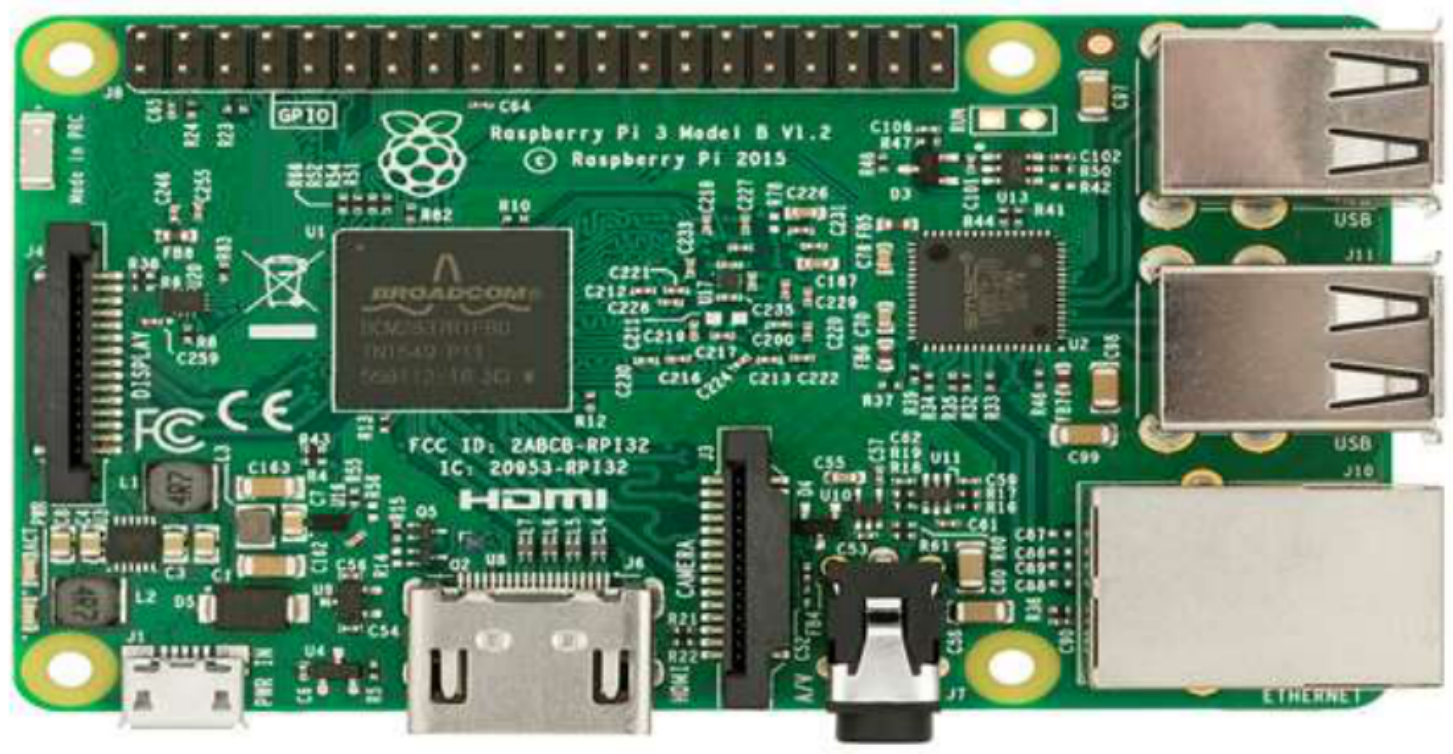

Figure 1 Raspberry pi

The latest release is Raspberry pi 3 (inbuilt wifi and BLE). However, the Raspberry pi used in the project is model $2 \mathrm{~B}$. Relays are switches that open and close circuits electromechanically orelectronically. Relays control one electrical circuit by opening and closing contacts in another circuit. As relay diagrams show, when a relay contact is normally open (NO), there is an open contact when the relay is notenergized. When a relay contact is Normally Closed (NC), there is a closed contact when the relay is not energized. In either case, applying electrical current to the contacts will change their state. Relays are generally used to switch smaller currents in a control circuit and do not usually control power consuming devices except for small motors and Solenoids that draw low amps. Nonetheless, relays can "control" larger voltages and amperes by having an amplifying effect because a small voltage applied to a relays coil can result in a large voltage being switched by the contacts . Protective relays can prevent equipment damage by detecting electrical abnormalities, including over current, undercurrent, overloads and reverse currents. In addition, relays are also widely used to switch starting coils, heating elements, pilot lights and audible alarms.We Used to provide wireless internet access. Not to be used with raspberry pi 3 as it has built in wifi module. Now we Connect the sound card to any of the USB port of raspberry pi and ensure its connection using the command: lsusb, check whether the device has been recognized.

\section{SOFTWARE REQUIRED PYTHON}

Python is an interpreter, object-oriented, high-level programming language with dynamic semantics. Its high-level built in data structures, combined with dynamic typing and dynamic binding, make it very attractive for Rapid Application Development, as well as for use as a scripting or glue language to connect existing components together. Python's simple, easy to learn syntax emphasizes readability and therefore reduces the cost of program maintenance. Python supports modules and packages, which encourages program modularity and code reuse. The Python interpreter and the extensive standard library are available in source or binary form without charge for all major platforms, and can be freely distributed. 


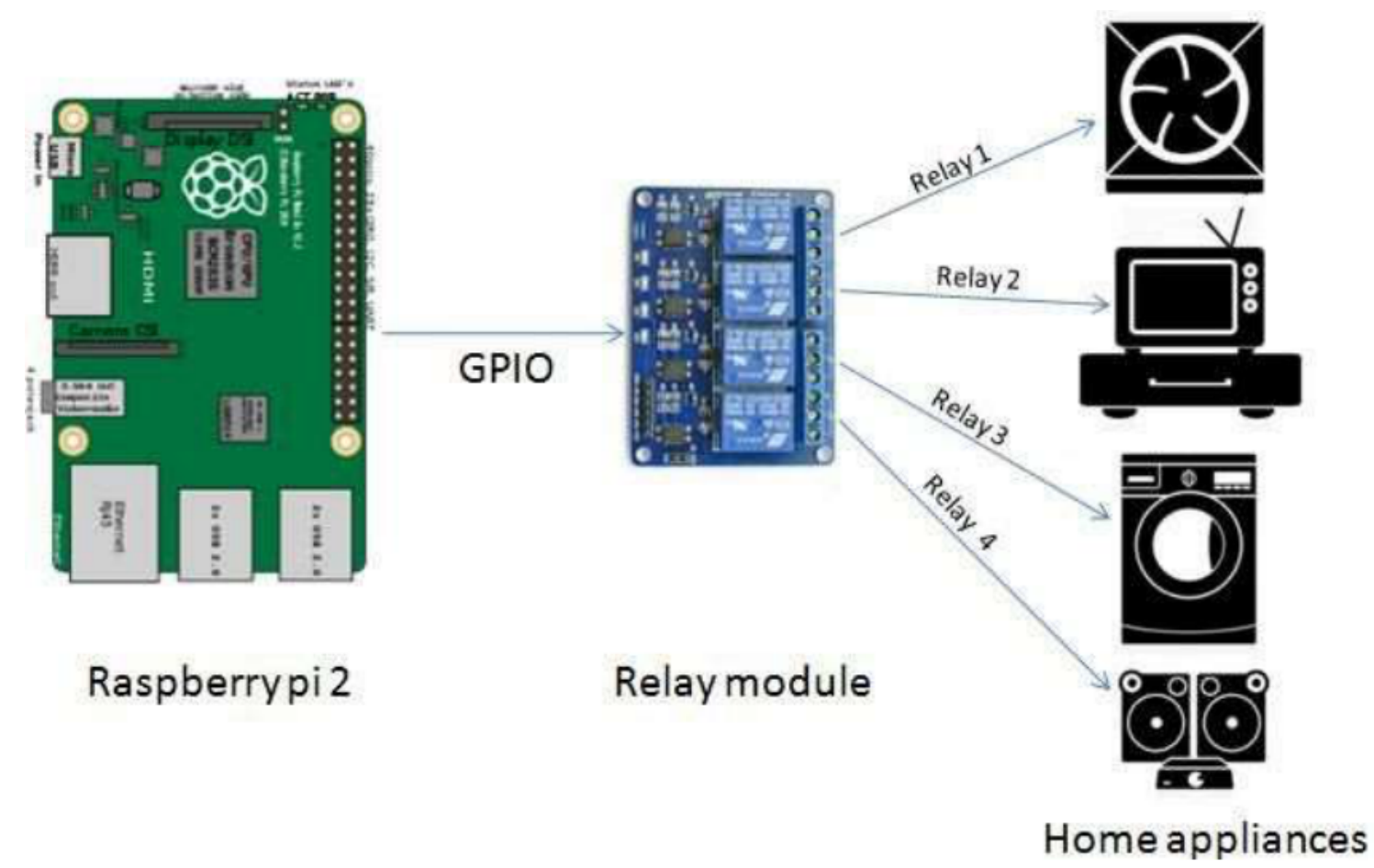

\section{Speech Recognition}

The speech recognition uses a python script called speechrecognition.py. This python script uses speech recognition library to record and process speech. It uses the wit API online Speech-To-Text platform to convert the speech to text. When the user provides the speech through the microphone the speech gets recorded by the python script in WAV format and is send to the wit platform along with an unique API key provided by the wit account. Once the audio file reaches wit it converts it in to text using their advanced speech recognition algorithm. The text is then send back to the python script[1].In the python script it is checked with the predefined commands to turn appliances on or off. If the phrase matches with text received then corresponding string (ON or OFF) is written to the file of respective relay. A python script is used called iot.py. It monitors the content of the files for each relays (relay1.txt - relay4.txt). Based on the content of the file ie either "ON" or "OFF" the python scripts turns the GPIO pin high or low for each of the relay connected to it.

\section{Web Interface}

The Raspberry pi hosts the web pages to control devices through an Apache web server. The web pages include index.html, controller.php, button.php, checklogin.php, logout.php. There file defined for each relays containing the string "ON" or "OFF". The index page acts as the home page. Once user logs in with a username and password the checklogin page checks the credential with a preset credential [3]. If a match occurs user will be redirected to controller page with after setting a session variable else user will be redirected to login page. In the controller page user can switch device on or off through buttons. On press of each button a button page is invoked which checks the button id and writes appropriate string to the corresponding relay file. A python script is used called iot.py. It monitors the content of the files for each relays (relay1.txt - relay4.txt). Based on the content of the file ie either "ON" or "OFF" the python scripts turns the GPIO pin high or low for each of the relay connected to it.Thus the relay turns on and off based on user input and any appliance can be connected to it to get controlled via internet. [2] 


\section{RESULT ANALYSIS Hardware Implementation}

To make the system hardware we gone through below block diagram. The whole block diagram is divided into two sections, first is Server side and other one is client or user side.

Server side is totally installed on the Raspberry pi. Server is created on the Raspberry pi with the help of LAMP (Linux, Apache, MySQL, PHP). Two PHP files are created and stored on the Server that we have created on the Raspberry pi. Raspberry pi is having 40 GPIO pins. These pins are used to control the home appliances [4]. Relay are connected to the GPIO pins of the Raspberry pi through the Relay Driver Circuitry. Output of the GPIO pins is 3.3V. In order to drive Relay minimum $6 \mathrm{~V}$ voltage is required so this can be obtained with the help of Relay driver circuitry. All home appliances are connected to the Relay. Client side is nothing but a User side. Users need to use Mobile device to access the Raspberry pi through the internet. Once the user connects mobile device in network and after putting the IP address of the Raspberry pi in the browser of Mobile device will be able to see the web page which contains UI to control home appliances in each room. UI simply shows the number of rooms and home appliances present in each room. It also contains buttons to toggle the status of home appliances of each room. Number of home appliances can be controlled simultaneously.

\section{Software Implementation}

To implement the entire process we made Programming by using PHP language. Two PHP files are created. One is index. php and another one is switch Device. php. These two files are stored on the local LAMP server of the Raspberry pi. Dreamweaver software is used to develop the web page and to create the UI present on that web page

\section{CONCLUSION}

Indeed, we can easily install a variety of sophisticated home automation systems to make your life simpler. Whether we choose to incorporate just a few features like lighting and door sensors or invest in all the options described above, smart home systems serve three main benefits: security, accessibility and most of all, simplicity.

\section{REFERENCES}

[1] D. Norris, the Internet of Things: Do-It-Yourself at Home Projects for Arduino, Raspberry Pi and BeagleBone Black. Tab Electronics, 2015.

[2] D. Giusto, A. Iera, G. Morabito, and L. Atzori, The Internet of Things. New York, NY: Springer New York, 2010.

[3] Raspberry pi as a sensor Web node for home automation Vladimir Vujovic, Mirjana Maksimovic http://dx.doi.org/10.1016/j.compeleceng.2015.01 .019

[4] A. Z. Alkar and U. Buhur, "An internet based wireless home automation system for multifunctional devices," IEEE Trans. Consum. Electron., vol. 51, no. 4, pp. 1169-1174, Nov. 2005.

[5] Jump up Bush, Steve (25 May 2011). "Dongle computer lets kids discover programming on a TV". Electronics Weekly. Retrieved 11 July, 2011

[6] Horan B. Practical Raspberry Pi. USA: Apress; 2013. 